กORTH CLIn ISTANBUL 2015;2(3):189-195

doi: $10.14744 /$ nci.2015.79188

\title{
Prognosis of patients in a medical intensive care unit
}

\author{
Ali Ugur Unal, ${ }^{1}$ Osman Kostek, ${ }^{1}$ Mumtaz Takir, ${ }^{2}$ Ozge Caklili, ${ }^{1}$ Mehmet Uzunlulu, ${ }^{1}$ Aytekin Oguz ${ }^{1}$ \\ ${ }^{1}$ Department of Internal Medicine, Istanbul Medeniyet University, Goztepe Training and Research Hospital, Istanbul, Turkey \\ 2Division of Endocrinology and Department of Internal Medicine, Istanbul Medeniyet University, Goztepe Training and Research \\ Hospital, Istanbul, Turkey
}

\begin{abstract}
OBJECTIVE: The aim of this study is to evaluate the demographic characteristics of critically ill patients and to determine intensive care unit (ICU) mortality and its predictors.

METHODS: This study was undertaken in the Istanbul Medeniyet University Göztepe Training and Research Hospital Medical ICU. Between May 2012 and January 2013, 111 patients (53 female, 58 male; mean age, $73.79 \pm 14.73$, mean length of ICU length stay, $9.1 \pm 10.7$; prevalence of geriatric patients, $77.5 \%$ ) were admitted to the ICU. The common indications for ICU admission, prevalence of mechanical ventilation support, hematological and biochemical parameters and their effects on mortality were assessed.
\end{abstract}

RESULTS: The common indications for ICU admission were hemodynamic instability (48.6\%), respiratory failure (27.9\%) and sepsis (15.3\%). Hypertension (46.8\%) was the most common comorbidity. Prevalance rates of heart failure and diabetes mellitus were $32.4 \%$ and $25.2 \%$ respectively. Mortality rate was $52.3 \%$ in all patients. Approximately $80 \%$ of all deaths was observed within the first fifteen-day. In additon, mortality rate (85.7\%) was prominent within patients in need of the mechanical ventilation support. Mechanical ventilation requirement, increased ferritin and vitamin B12 levels were independent risk factors for mortality in critically ill patients ( $<<0.01$, for all).

CONCLUSION: Mortality rate was higher in medical ICU. Herein, increased prevalence of geriatric population, concomitant comorbidities and mechanical ventilation requirements may play role.

Keywords: Comorbidity; mechanical ventilation; medical intensive care unit; morbidity.

$\mathrm{I}$ ntensive care units (ICUs) are special treatment units managed by specially trained health personnel, and designed for the monitorization, and treatment of life-threatening organ failures which can be seen during the course of acute, and chronic diseases [1]. Most of the patients are monitored while intubated, or in some cases after extubation. Some neurological diseases (Alzheimer, stroke, cerebral palsy etc.), and life-threatening complications of respiratory tract infections, intoxications, and sepsis are frequently monitored in intensive care units $[2,3]$. In intensive care units all-cause

Received: January 30, 2015 Accepted: December 29, 2015 Online:

Correspondence: Dr. Mumtaz TAKIR. Istanbul Medeniyet Universitesi Goztepe Egitim ve Arastirma Hastanesi, Endokrinoloji ve Metabolizma Klinigi, 34710 Istanbul, Turkey.

Tel: +90 216 - 5709195 e-mail: mumtaztakir@yahoo.com

(c) Copyright 2015 by Istanbul Northern Anatolian Association of Public Hospitals - Available online at www.kuzeyklinikleri.com 
mortality rates are higher relative to other services. Age, primary disease, the severity of disease-related complications can be among causative factors [4]. In addition to these factors the association of mortality with acute phase reactants as $\mathrm{C}$-reactive protein, albumin, and ferritin has been reported [5-7].

Our aim was to determine demographic characteristics of patients hospitalized in intensive care unit of the department of internal medicine, and also mortality rates, and prognostic parametres on mortality.

\section{MATERIALS AND METHODS}

A total of 111 patients aged $>18$ years with their medical files who were hospitalized in intensive care unit of Department of Internal Medicine of Göztepe Training and Research Hospital from the May 2nd, 2012 (inauguration date of ICU) up to January 1st, 2013 were retrospectively examined. The investigation was conducted according to the principles outlined in the Declaration of Helsinki. The study protocol was approved by Göztepe Training and Research Hospital Ethics Committee (29/H-12/28/2012).

Study design: From patient registration forms, information about age, gender, diagnosis at admission to the intensive care unit, length of ICU stay, requirement for, and duration of mechanical ventilation, clinical, and laboratory data, and outcomes of the patients (ratio of ICU survivors and nonICU survivors patients) were recorded. Diagnoses of admission were retrieved from patient files, and examined based on their ICD codes. Epicrises were scrutinized in detail, and associated comorbidities (chronic obstructive pulmonary disease, diabetes mellitus, congestive heart failure, hypertension, chronic renal failure) were recorded. Admision diagnoses of the patients were investigated in consideration of their ICD codes indicated in their medical files. Associated comorbidities indicated in their detailed epicrises (chronic obstructive pulmonary disease, diabetes mellitus, congestive heart failure, hypertension, chronic renal failure) were recorded. Admission diagnoses were categorized as hemodynamic instability (systolic blood pressure $<90$ $\mathrm{mmHg}$, heart rate $>120 / \mathrm{min}$ due to any cause apart from sepsis, acute or chronic respiratory failure. sep- sis, and respiratory failure.

Laboratory parameters: Data related to whole blood counts, biochemical test results (urea, creatinine, uric acid, sodium, potassium, calcium, phosphorus, AST, ALT, total protein, albumin, CRP, BNP, sedimentation rate, vitamin B12, folat, ferritin, iron, total iron binding capacity, transferrin saturation, INR, procalcitonin) were recorded. In all patients, all-cause mortality rates, diagnosis at admission, length of hospital stay, incidence of comorbidities, number (\%) of patients who received invasive mechanical ventilation, hematological, and biochemical data, and their correlations with mortality were evaluated. The patients were categorized as those with duration of hospitalization shorter or longer than 15 days, and compared based on age, indication for hospitalization, presence of comorbidities, requirement for mechanic ventilation, and mortality rates.

Statistical analysis: Statistical analysis was performed using SPSS for Windows V.21.0 program. Normality of distribution of variables was checked by using Kolmogorov Smirnov test. Continuous variables were expressed as mean $\pm \mathrm{SD}$. Classifiable data were analyzed using chi-square test, numerical data with Mann-Whitney $U$ test. Independent factors predicting mortality were demonstrated using logistic regression (backward stepwise, LR) test, level of significance, estimated relative risk (odds ratio-OR), and $95 \%$ confidence $\mathrm{CI}$ ) interval. Statistical significance was set at a level of $p<0.05$ within $95 \%$ confidence interval.

\section{RESULTS}

Mean age of the study participants was $73.79 \pm 14.73$ years (min-max:21-93) with a similar gender distribution ( $p>0.05)$. Geriatric patients ( $>65$ years) consisted $77.5 \%(n=86)$ of ICU population. The associated comorbidities in order of decreasing frequency were as follows: hypertension, congestive heart failure, diabetes mellitus, chronic obstructive pulmonary disease, and chronic renal failure. (Table 1). The patients were most frequently hospitalized in ICU with indications of hemodynamic disorder (48.6\%), respiratory failure (27.9\%), and sepsis (15.3\%). The patients were mostly referred to ICU from inpatient services 


\begin{tabular}{|c|c|c|}
\hline & $\mathrm{n}$ & $\%$ \\
\hline Age, years (mean $\pm S D)$ & \multicolumn{2}{|c|}{$73.79 \pm 14.73$} \\
\hline \multicolumn{3}{|l|}{ Gender } \\
\hline Female & 53 & 47.7 \\
\hline Male & 58 & 52.3 \\
\hline \multicolumn{3}{|l|}{ Comorbidities } \\
\hline Hypertension & 52 & 46.8 \\
\hline Congestive heart failure & 36 & 32.4 \\
\hline Diabetes mellitus & 28 & 25.2 \\
\hline Chronic obstructive pulmonary disease & 17 & 15.3 \\
\hline Chronic renal failure & 16 & 14.4 \\
\hline
\end{tabular}

SD: Standard deviation.

(79.3\%) followed by direct referrals from the emergency service (18.9\%), and an external center (1.8\%).

All-cause mortality rate among patients hospitalized in ICU was $52.3 \%$, and $79.3 \%$ of deaths occurred within the first 15 days of hospitalization. All-cause mortality rate among patients receiving IMV support was 85.7 percent.

Less than half of the ICU patients (44.1\%) required mechanical ventilation. Distribution of these patients according to their indications for hospitaliza-

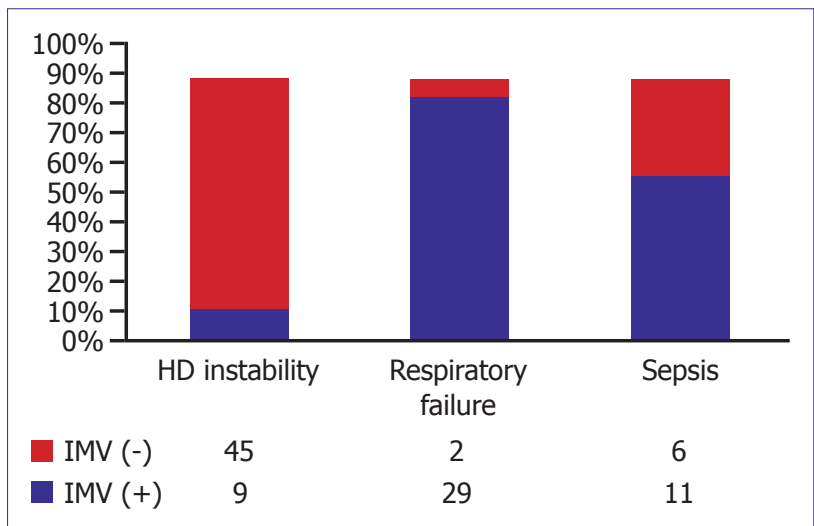

FIGURE 1. Frequency of invasive mechanical ventilation (IMV) use among intensive care unit patients based on their diagnoses of admission.

tion is given in Figure 1. Most (93.5\%) of the patients with respiratory distress, $64.7 \%$ of those with sepsis, and $16.6 \%$ of the individuals with hemodynamic instability required invasive mechanical ventilation.

Median duration of hospitalization was 9.1 (min-max: 1-49) days. Some (15.3\%) patients were hospitalized for more than 15 days. Hemodynamic instability was more frequently seen in patients hospitalized for more than 15 days $(p=0.003)$, while respiratory failure, and requirement for mechanical ventilation were more often encountered in these patients $(p=0.01$, and $p=0.002$, respectively) (Table 2 ).

TABLE2. Factors effective on the length of intensive care unit stay

\begin{tabular}{llll}
$\frac{\leq 15 \text { days }(n=85)}{n}$ & & & \multicolumn{2}{c}{$>15$ days $(n=17)$} \\
& $\%$ & $n$ & $\%$
\end{tabular}

\begin{tabular}{lccccc}
\hline Age, year (mean \pm SD) & \multicolumn{2}{c}{$72.3 \pm 14.9$} & \multicolumn{2}{c}{$77.3 \pm 15.7$} & 0.21 \\
Hemodynamic instability & 49 & 57.6 & 3 & 17.6 & $\mathbf{0 . 0 0 3}$ \\
Respiratory failure & 19 & 22.4 & 12 & 70.6 & $\mathbf{0 . 0 1}$ \\
Sepsis & 14 & 16.5 & 2 & 11.8 & 0.62 \\
Chronic obstructive pulmonary disease & 13 & 15.3 & 4 & 23.5 & 0.4 \\
Diabetes mellitus & 26 & 30.6 & 2 & 11.8 & 0.11 \\
Congestive heart failure & 32 & 37.6 & 4 & 23.5 & 0.26 \\
Chronic renal failure & 13 & 15.3 & 3 & 17.6 & 0.8 \\
Invasive mechanical ventilation & 35 & 41.2 & 14 & 82.4 & $\mathbf{0 . 0 0 2}$ \\
Mortality & 46 & 54.1 & 12 & 70.6 & 0.21 \\
\hline
\end{tabular}

SD: Standard deviation. 


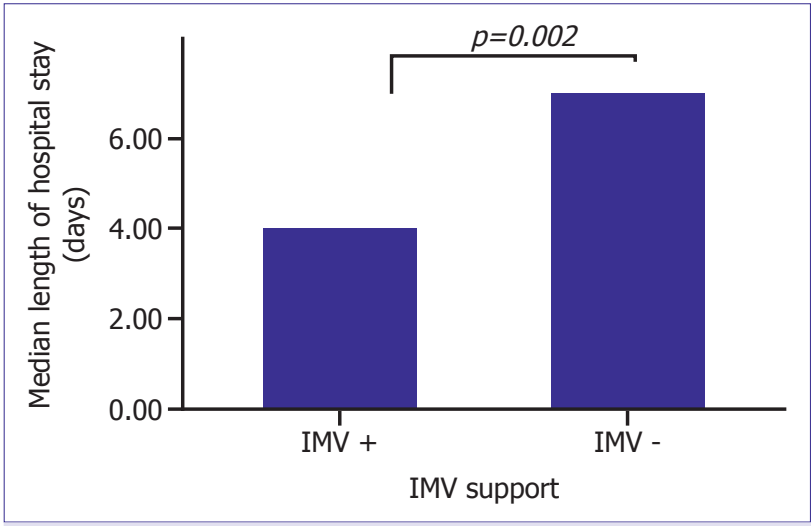

FIGURE 2. Comparison of median lengths of hospital stays in patients who received, and did not receive invasive mechanic ventilation support.
The correlation between IMV, and length of ICU stay is presented in Figure 2. Median duration of hospitalization among patients who received, and did not receive IMV support was 7 (IQR $2-18$ ), and 4 (IQR 2-8) days, respectively. As is seen, IMV support prolonged hospital stay of the patients $(p=0.002)$.

Biochemical, and hematological data of the study participants are given in Table 3. When compared with ICU survivors; higher serum urea, creatinine, total bilirubin, ferritin, vitamin B12 levels (for all $\mathrm{p}<0.05$ ) and lower total protein, and albumin levels (for both $\mathrm{p}<0.05$ ) were detected in nonICU survivors.

TABLE 3. Biochemical parametres among patients hospitalized in the intensive care unit

\begin{tabular}{|c|c|c|c|c|}
\hline & All patients & Non-ICU Survivors & Survivors & $\mathrm{p}$ \\
\hline & Mean \pm SD & Mean \pm SD & Mean \pm SD & \\
\hline Hemoglobin $(n=102)$ & $9.74 \pm 1.85$ & $9.44 \pm 1.7$ & $9.98 \pm 1.9$ & $>0.05$ \\
\hline Hemotocrit $(n=102)$ & $30.2 \pm 5.6$ & $29.23 \pm 5.3$ & $30.99 \pm 6.0$ & $>0.05$ \\
\hline Platelet $(n=102)$ & $203.8 \pm 147.7$ & $187.39 \pm 159.5$ & $219.52 \pm 114.5$ & $>0.05$ \\
\hline MPV $(n=102)$ & $10.3 \pm 2.0$ & $10.63 \pm 2.5$ & $9.93 \pm 1.2$ & $>0.05$ \\
\hline Leukocyte $(n=102)$ & $12.6 \pm 8.0$ & $13.45 \pm 9.3$ & $11.54 \pm 6$ & $>0.05$ \\
\hline Ferritin $(n=102)$ & & & & \\
\hline Median & 238 & 494 & 143 & $<0.05$ \\
\hline IQR & $95-655$ & $116-903$ & $85-259$ & \\
\hline Sedimentation rate $(n=95)$ & $55.32 \pm 32.2$ & $55.95 \pm 33.8$ & $54.36 \pm 29.8$ & $>0.05$ \\
\hline $\operatorname{CRP}(n=111)$ & $12.27 \pm 12.7$ & $14.35 \pm 15$ & $10.26 \pm 9.8$ & $>0.05$ \\
\hline Folic acid $(n=101)$ & $6.36 \pm 4.4$ & $6.05 \pm 4.7$ & $7.08 \pm 4.4$ & $>0.05$ \\
\hline Vitamin B12 $(n=102)$ & & & & \\
\hline Median & 345 & 505 & 198 & $<0.05$ \\
\hline IQR & $182-719$ & 241-1094 & $139-364$ & \\
\hline Glucose $(n=102)$ & $152.9 \pm 101.3$ & $148.08 \pm 114$ & $155.90 \pm 88.4$ & $>0.05$ \\
\hline Urea $(n=110)$ & $102.8 \pm 64.7$ & $120.47 \pm 73$ & $77.95 \pm 45.9$ & $<0.05$ \\
\hline Uric acid $(n=110)$ & $8.1 \pm 11.6$ & $7.47 \pm 8.3$ & $8.87 \pm 15.4$ & $>0.05$ \\
\hline Creatinine $(n=110)$ & $2.1 \pm 1.7$ & $2.38 \pm 1.8$ & $1.56 \pm 1.32$ & $<0.05$ \\
\hline Sodium $(n=110)$ & $139.4 \pm 8.8$ & $139.59 \pm 10$ & $139.52 \pm 7.5$ & $>0.05$ \\
\hline Potassium $(n=110)$ & $4.1 \pm 0.9$ & $4.06 \pm 0.9$ & $4.21 \pm 0.92$ & $>0.05$ \\
\hline T. bilirubin $(n=104)$ & $11.1 \pm 17.9$ & $14.26 \pm 20$ & $8.27 \pm 14.8$ & $<0.05$ \\
\hline GGT $(n=103)$ & $61.8 \pm 70.9$ & $76.38 \pm 82.9$ & $49.59 \pm 56.5$ & $>0.05$ \\
\hline Protein $(n=98)$ & $5.78 \pm 30$ & $5.49 \pm 1.03$ & $6.08 \pm 0.9$ & $<0.05$ \\
\hline Albumin $(n=99)$ & $2.90 \pm 0.98$ & $2.58 \pm 0.74$ & $3.20 \pm 0.68$ & $<0.05$ \\
\hline $\operatorname{HbA1c}(n=45)$ & $6.35 \pm 1.5$ & $6.06 \pm 1$ & $6.77 \pm 2.06$ & $>0.05$ \\
\hline
\end{tabular}

SD: Standard deviation; MPV: Mean platelet volume IQR: Interquartile range; CRP: C-reactive protein; GGT: Gamma glutamyl transferase. 
TABLE 4. Factors affecting mortality rates of the ICU inpatients

\begin{tabular}{lccc} 
& $\mathrm{B}$ & OR $(95 \% \mathrm{CI})$ & $\mathrm{p}$ \\
\hline Age & 1.048 & $1.000-1.098$ & 0.052 \\
Invasive mechanical & & & \\
ventilation & 18.462 & $4.750-71.760$ & $\mathbf{< 0 . 0 0 1}$ \\
Ferritin & 1.002 & $1.000-1.004$ & $\mathbf{0 . 0 2 8}$ \\
Vitamin B12 & 1.002 & $1.001-1.004$ & $\mathbf{0 . 0 0 7}$
\end{tabular}

ICU: Intensive care units; OR: Odds ratio.

TABLE 5. Vitamin B12, and Ferritin values based on the area under ROC curve analysis

\begin{tabular}{lcccc} 
& AUC & SE & $95 \%$ CI & $p$ \\
\hline Vitamin B12 & 0.732 & 0.053 & $0.629-0.835$ & $<0.001$ \\
Ferritin & 0.712 & 0.053 & $0.608-0.815$ & $<0.001$ \\
\hline
\end{tabular}

AUC: Area under the curve; SE: Standard error.

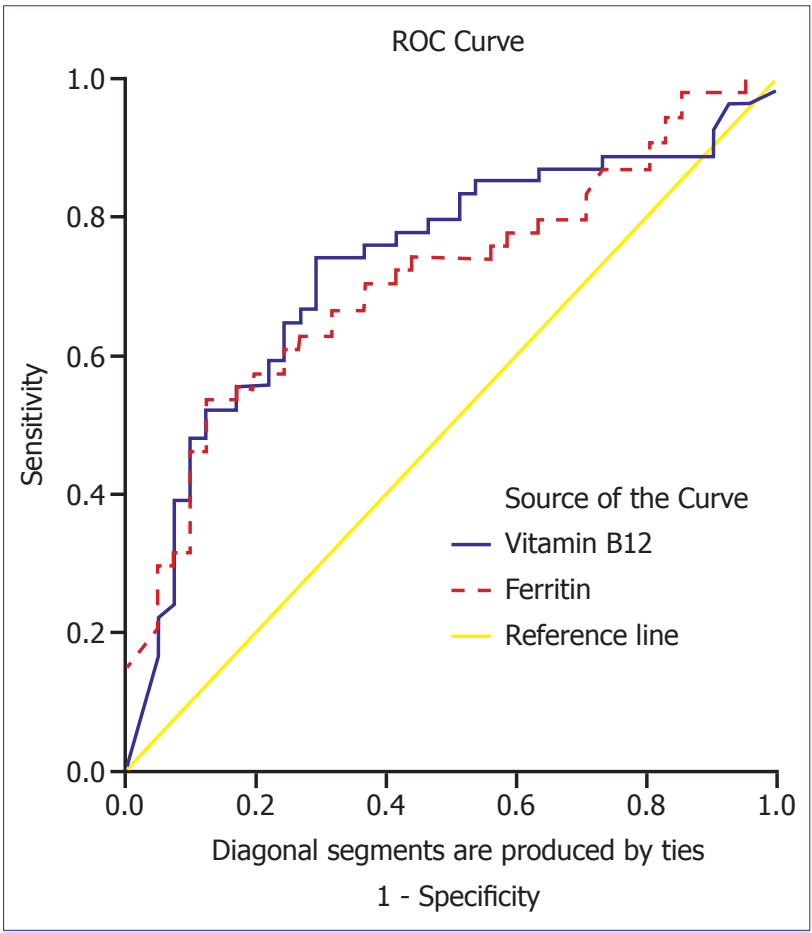

FIGURE 3. Factors effecting mortality rates in the intensive care unit.

TABLE 6. Cut-off values of vitamin B12, and ferritin levels for the prediction of mortality rates

\begin{tabular}{lccccc} 
& Sensitivity (\%) & Specificity (\%) & PPD (\%) & NPD (\%) & Accuracy (\%) \\
\hline Vitamin B12 ( $\geq 282 \mathrm{mg} / \mathrm{dL})$ & 74 & 70.7 & 76.9 & 67.4 & 72.6 \\
Ferritin (212 mg/dL) & 70.3 & 63.4 & 71.6 & 60.4 & 67.3 \\
\hline
\end{tabular}

PPD: Positive cut-off value; NPD: Negative cut-off value.

Independent predictors of mortality were requirement for IMV, higher ferritin, and vitamin B12 levels (Tables 4, 5; Figure 3). The best cut-off value of vitamin B12 was $282 \mathrm{mg} / \mathrm{dL}$ which predicted mortality with $74 \%$ sensitivity, and $70.7 \%$ specificity. On the other hand, the best cut-off value of ferritin was $212 \mathrm{mg} / \mathrm{dL}$ which predicted mortality with $70.3 \%$ sensitivity, and $63.4 \%$ specificity (Table 6 ).

\section{DISCUSSION}

In this study, higher all-cause mortality rates in patients hospitalized in intensive care unit were observed which might be attributed to increased number of geriatric patients consulting to ICUs, associated comorbidities, and frequent need for IMV.

Elder patients hospitalized in the intensive care unit constitute privileged, and very complex patient group. Generally elder patients are hospitalized because of acute exacerbations of underlying chronic health problems or multiorgan disorders. As reported in many literature studies $46 \%$ of the patients hospitalized in ICUs are elder people [4]. In patients hospitalized in ICU a significant increase in all-cause mortality rates have been observed $[8,9$, $10,11]$. In our study, mean age of the patients hospitalized in ICU was $73.9 \pm 14.7$ years, and $77.5 \%$ of all patients were in the geriatric age (>65) group. 
Uysal et al, reported an overall ICU mortality rate of 43 percent [4]. However in our study overall ICU mortality rate was 52.3 percent. In an international multi-centered prospective study $(n=15.757)$ mechanical ventilator had been used in $33 \%$ of the cases with a mean duration of $5.9 \pm 7.2$ days. Besides average ICU mortality rate was reported as $31 \%$ among patients who had received mechanical ventilation therapy for more than 12 hours [12]. In our study, mechanical ventilator was used in $44.1 \%$ of the patients for a mean period of $12.7 \pm 13.5$ days, ICU mortality rate among mechanical ventilator users was 85.7 percent.

Altray et al. performed a study to determine factors related to mortality rates in ICU patients, and observed that presence of arrhytmia, requirement for cardiotonic drugs, need for mechanical ventilation, and its duration had independent effects on all-cause mortality [13]. In our study, we also determined the impact of mechanical ventilation on mortality per se.

Higher ferritin levels may stem from acute phase reactions regulated by cytokines [14]. In a study by Rogers et al. the authors had demonstrated that interleukin-1 (IL-1) accelerates transcription process of ferritin [15]. In our study, higher ferritin values were detected as one of the independent factors which had an impact on mortality rates. Higher ferritin levels may be associated with significant clinical conditions, and from this perspective it can be accepted as a marker of a nonspecific disease.

Very scarce number of studies have been performed on higher vitamin B12 values. In a study performed by Andrès $E$ et al. the authors indicated that higher vitamin B levels can be paradoxically seen in cases with functional cobalamine insufficiency, and added that its elevated levels can be detected in cases with severe disease states as a diagnostic marker of prognosis at an early stage of the disease [16]. In our study, the patients did not receive vitamin B12 treatment during their ICU stay or previously as disclosed from patient files. Statistically significantly higher vitamin B12 levels were detected in non-ICU survivors patients who had not received vitamin B12 replacement when compared with those ICU survivors.
While evaluating the outcomes of our study, some limitations should be considered. Relatively lower number of our patient population because of recent inauguration of our intensive care unit, inability to apply prognostic scoring systems as APACHE (Acute Physiology and Chronic Health Evaluation), Glasgow coma, retrospective retrieval of patient data from hospital registration system, and missing information in patient data charts are limitations of our study.

In conclusion, increased number of geriatric patients, and frequent requirement of IMV might have a role in higher all-cause mortality rates detected in the intensive care unit of the department of internal medicine. In addition, among predictors of mortality, higher vitamin B12, and ferritin levels are remarkable. Therefore, larger-scale studies which will demonstrate the correlation between vitamin B12, and ferritin levels and mortality in critically ill patients should be performed.

Conflict of Interest: No conflict of interest was declared by the authors.

Financial Disclosure: The authors declared that this study has received no financial support.

\section{REFERENCES}

1. Brilli RJ, Spevetz A, Branson RD, Campbell GM, Cohen H, Dasta JF, et al. Critical care delivery in the intensive care unit: defining clinical roles and the best practice model. Crit Care Med 2001;29:2007-19.

2. Tran DD, Groeneveld AB, van der Meulen J, Nauta JJ, Strack van Schijndel RJ, Thijs LG. Age, chronic disease, sepsis, organ system failure, and mortality in a medical intensive care unit. Crit Care Med. 1990;18:474-9.

3. Zimmerman JE, Kramer AA, Knaus WA. Changes in hospital mortality for United States intensive care unit admissions from 1988 to 2012. Crit Care 2013;17:R81.

4. Uysal N, Gündoğdu N, Börekçi Ş, Dikensoy Ö, Bayram N, Uyar $\mathrm{M}$, et al. Prognosis of Patients in a Medical Intensive Care Unit of a Tertiary Care Centre. Yoğun Bakım Derg 2010;1:1-5.

5. Pasceri V, Willerson JT, Yeh ET. Direct proinflammatory effect of $\mathrm{C}$-reactive protein on human endothelial cells. Circulation 2000;102:2165-8.

6. Nicholson JP, Wolmarans MR, Park GR. The role of albumin in critical illness. Br J Anaesth 2000;85:599-610.

7. Jehn ML, Guallar E, Clark JM, Couper D, Duncan BB, Ballantyne CM, et al. A prospective study of plasma ferritin level 
and incident diabetes: the Atherosclerosis Risk in Communities (ARIC) Study. Am J Epidemiol 2007;165:1047-54.

8. Trivedi TH, Shejale SB, Yeolekar ME. Nosocomial pneumonia in medical intensive care unit. J Assoc Physicians India 2000;48:1070-3.

9. Seferian EG, Afessa B. Adult intensive care unit use at the end of life: a population-based study. Mayo Clin Proc 2006;81:896-901.

10. Scott BH, Seifert FC, Grimson R, Glass PS. Octogenarians undergoing coronary artery bypass graft surgery: resource utilization, postoperative mortality, and morbidity. J Cardiothorac Vasc Anesth 2005;19:583-8.

11. Ray DC, Drummond GB, Wilkinson E, Beckett GJ. Relationship of admission thyroid function tests to outcome in critical illness. Anaesthesia 1995;50:1022-5.

12. Esteban A, Anzueto A, Frutos F, Alía I, Brochard L, Stewart
TE,et al. Characteristics and outcomes in adult patients receiving mechanical ventilation: a 28-day international study. JAMA 2002;287:345-55.

13. Altıay G, Tabakoğlu E., Özdemir L, Tokuç B, Çevirme L, Hatipoğlu ON, et al. Mortality Rates and Related Factors in Respiratory Intensive Care Unit Patients. Toraks Dergisi 2007;8:79-84.

14. Lee MH, Means RT Jr. Extremely elevated serum ferritin levels in a university hospital: associated diseases and clinical significance. Am J Med 1995;98:566-71.

15. Rogers J, Durnowicz G, Kasschau K, Lacroix L, Bridges K. A motif within tha 5 ' non-coding regions of acute phase $\mathrm{mRna}$ mediates control of ferritin translation by IL-1B and may contribute to the anemia of chronic disease. Blood 1991;78:361.

16. Andrès E, Serraj K, Zhu J, Vermorken AJ. The pathophysiology of elevated vitamin B12 in clinical practice. QJM 2013;106:505-15. 